\title{
A genome-wide association study identified genetic loci for end-stage liver disease in the Korean population
}

\author{
Hye-Mi Jang ${ }^{1, \star}$, Dong Jin Joo ${ }^{2, \star}$, Sung Min Kim, Hyun-Young Park ${ }^{3}$, Bong-Jo Kim, Myoung Soo Kim², Young Jin Kim \\ ${ }^{1}$ Division of Genome Science, Korea National Institute of Health, Cheongwon, Korea \\ ${ }^{2}$ Department of Surgery, Yonsei University College of Medicine, Seoul, Korea \\ ${ }^{3}$ Department of Precision Medicine, Korea National Institute of Health, Cheongwon, Korea
}

Background: Liver transplantation (LT) is the most effective treatment for patients with end-stage liver diseases. To understand the contribution of genetic factors for liver diseases, several genome-wide association studies (GWAS) have been conducted. However, most of studies were conducted in Europeans. In 2014, a prospective designed version of the Korean Organ Transplantation Registry (KOTRY) was launched, supported by Korea National Institute of Health (KNIH). For GWAS on transplantation, $\mathrm{KNIH}$ is producing more than 9,000 KOTRY samples of genome data using Korea Biobank Array (KBA), a fully customized Single nucleotide polymorphisms (SNP) microarray for Koreans. In this study, we performed a GWAS to identify loci associated with LT in the Korean population.

Methods: All samples of cases and controls were genotyped using KBA. After quality control, there were 2,082 samples of LT cases from the KOTRY and 54,354 controls from the Korean Genome and Epidemiology Study (KoGES). Controls were selected as samples without liver diseases based on self-report survey data. Variants with minor allele frequency $\geq 1 \%$ were selected and imputed using IMPUTE v4. Logistic regression was performed by adjusting age and gender using EPACTS software.

Results: At $\mathrm{P}<5 \times 10^{-8}$, four loci including ADH1B, HLA-DPB1, ALDH2, and PNPLA3 were associated with LT. All loci were previously associated with cirrhosis and biliary cholangitis. Among them, a missense variant rs671 at ALDH2 was previously associated with hypertension, diabetes, cardiovascular diseases, alcohol dependence, liver enzymes, and lipid traits.

Conclusions: We performed GWAS on LT in Koreans and validated previously reported four liver disease related loci including ADH1B, HLA-DPB1, ALDH2, and PNPLA3. However, these loci explained only small proportion of heritability. Therefore, LT GWAS with extended sample sizes is warranted to discover hidden genetic factors.

Corresponding author: Young Jin Kim

E-mail: anwltlarkr@gmail.com

Co-Corresponding author: Myoung Soo Kim

E-mail: YSMS91@yuhs.ac

*The authors contributed equally to this paper.

This work was supported by an intramural grant from the KNIH (2019-NG-054-01).

(c) The Korean Society for Transplantation

This is an Open Access article distributed under the terms of the Creative Commons Attribution Non-Commercial License (http://creativecommons.org/licenses/by-nc/4.0/) which permits unrestricted non-commercial use, distribution, and reproduction in any medium, provided the original work is properly cited. 